\title{
Monika Misztal $^{1}$, Magdalena Krakowska ${ }^{1}$, Monika Ryś-Bednarska ${ }^{1}$, Mariusz Śliwa ${ }^{1}$, Piotr Potemski ${ }^{1,2}$ (D)
}

${ }^{1}$ Copernicus Memorial Multidisciplinary Centre for Oncology and Traumatology, Lodz, Poland

${ }^{2}$ Chemotherapy Clinic, Oncology Department, Medical University of Lodz, Poland

\section{Symptoms of nervous system damage in a patient undergoing anti-PD1 immunotherapy}

\author{
Address for correspondence: \\ Lek. Monika Misztal \\ WWCOiT im. M. Kopernika w Łodzi \\ e-mail:misztalmon@gmail.com \\ Oncology in Clinical Practice \\ 2020, Vol. 16, No. 1, 34-35 \\ DOI: $10.5603 /$ OCP.2020.0006 \\ Translation: dr n. med. Dariusz Stencel \\ Copyright (C) 2020 Via Medica \\ ISSN 2450-1654
}

\begin{abstract}
Symptoms of nervous system damage during immunotherapy with anti-PD1 antibodies occur in approximately $6 \%$ of patients. The most commonly reported neurological adverse reactions are Guillain-Barre syndrome, polyneuropathy, demyelinating diseases, myasthenia gravis, and encephalitis.

In the presented patient with disseminated skin melanoma, after four administrations of nivolumab, paraesthesia hindering walking and weakness of the lower limbs appeared. Based on Doppler ultrasound, venous thrombosis was excluded. Computed tomography of the head did not show metastases or signs of intracranial ischaemia or bleeding. The patient was consulted neurologically several times. Steroid therapy, gabapentin, duloxetine, and painkillers were used. Despite the temporary improvement due to implemented treatment, the patient died. No definitive diagnosis could be made, but the symptoms suggest Guillain-Barre syndrome.

Key words: melanoma, nervous system damage, anti-PD1, nivolumab, side effects
\end{abstract}

Oncol Clin Pract 2020; 16, 1: 34-35

\section{Introduction}

Immunotherapy with checkpoint inhibitors is a valuable treatment for various cancers. With the more frequent use of this group of drugs, the frequency of observed side effects also increases. They result from excessive stimulation of the immune system. The most common symptoms affect the skin, digestive system, endocrine organs, and lungs. Neurological disorders and myocarditis are less commonly diagnosed [1]. Nervous system side effects affect approximately $6 \%$ of patients treated with anti-PD1 antibodies. The most commonly described are: Guillain-Barre syndrome, polyneuropathy, demyelinating diseases, myasthenia gravis, and encephalitis. Symptoms usually appear between 6 and 13 weeks of treatment [2]. The following is a description of a skin melanoma patient who experienced neurotoxicity symptoms during nivolumab treatment.

\section{Case report}

In May 2018, a 68-year-old patient diagnosed with melanoma of the chest skin with metastases to the right axillar and lung lymph nodes, without a mutation in the $B R A F$ V600 codon, began treatment with nivolumab at the Chemotherapy Clinic in Lodz. The patient was in a good general condition, did not report any complaints, and denied any comorbidities. The physical examination revealed a $7-\mathrm{cm}$ ulcerative skin tumour of the sternal area.

In July, after four antibody administrations, the patient reported paraesthesia in the lower extremities not responding to painkillers, and weakness in the right lower limb, which hindered walking. In laboratory tests and ultrasound Doppler of the lower limbs performed at that time, no abnormalities could be found that could explain the reported symptoms. Due to the severity of 
the symptoms, nivolumab treatment was discontinued and steroid therapy with prednisone $1 \mathrm{mg} / \mathrm{kg}$ was started, resulting in temporary improvement.

In August, the patient reported worsening of pain. Computed tomography of the head excluded metastases to the central nervous system, ischaemic stroke, and bleeding were. To assess the effectiveness of treatment, computed tomography of the chest, abdomen, and pelvis was performed, which showed the progression of the disease in the lungs and lymph nodes; the picture of the remaining organs remained normal. The patient was consulted neurologically, but apart from a slight paresis of the right lower limb, no abnormalities were found. According to the neurologist's recommendations, magnetic resonance imaging of the lumbosacral spine was planned and gabapentin and duloxetine were added. The prednisone dose was also increased to $2 \mathrm{mg} / \mathrm{kg}$. The drugs used once again allowed for a short-term improvement.

The ailments intensified again in September 2018. The man could barely get up from a chair. In addition, paraesthesia occurred in the upper limbs. The patient was referred to the neurological ward in which he died on October 1, 2018.

\section{Discussion}

Guillain-Barré syndrome is an acquired peripheral nerve disease of autoimmune aetiology. The main symptoms are paraesthesia and progressive paresis with abolition or weakness of deep reflexes. These ailments are usually of an ascending nature and are characterised by rapid growth over several days or weeks. The diag- nosis is based on the clinical picture and abnormalities in EMG (slowdown in conduction in the peripheral nerves) and cerebrospinal fluid test results (characteristic increase in protein concentration with normal cell number). Clinical signs usually precede the changes seen in EMG. In severe cases, with possible biting and swallowing disorders as well as respiratory and circulatory abnormalities, the treatment consists of securing basic life functions. Other patients use plasmapheresis or intravenous immunoglobulin preparations [3]. In the case of idiopathic Guillain-Barré syndrome, the use of glucocorticosteroids is not recommended; however, $1-2 \mathrm{mg} / \mathrm{kg}$ of prednisone is indicated in Guillain-Barré syndrome caused by anti-PD1 antibodies [2].

The wide spectrum of side effects of immunotherapy is a challenge for clinicians. In this patient, the symptoms were differentiated from a neurological disease not related to cancer and treatment, paraneoplastic syndrome, venous thrombosis, stroke, and disease progression in the form of central nervous system metastases. Despite the neurological consultation, no diagnosis could be established. As recommended, steroid therapy was used. These symptoms suggest Guillain-Barré syndrome.

\section{References}

1. Barquín-García A, Molina-Cerrillo J, Garrido P, et al. New oncologic emergencies: What is there to know about inmunotherapy and its potential side effects? Eur J Intern Med. 2019; 66: 1-8, doi: 10.1016/j. ejim.2019.05.020, indexed in Pubmed: 31130304.

2. Haanen J, Carbonnel F, Robert C, et al. Management of toxicities from immunotherapy: ESMO Clinical Practice Guidelines. Ann Oncol. 2017; 28(suppl 4): iv119-iv142.

3. Szczeklik A. Interna Szczeklika 2012 Podręcznik chorób wewnętrznych Kraków 2012. 